\title{
Gauge theory of pairing and spin fluctuations near the quantum critical point and superhigh-temperature superconductivity
}

\author{
J.R. Schrieffer \\ NHMFL and Department of Physics, Florida State University, Tallahassee, FL 32310 \\ E-mail: samaan@magnet.fsu.edu
}

Received September 9, 2005

\begin{abstract}
We develop a new theory of pairing and magnetic effect near the quantum critical point. Several novel properties are predicted: based on a spin fermion model, we derive two new interactions, i) a spin deformational potential $H_{s d p}$ proportional to the bandwidth $W$ (as opposed to the considerably smaller exchange coupling $J$ of the nearly antiferromagnetic Fermi liquid theory) and ii) a diamagnetic potential $H_{\text {dia }}$, quadratic in a gauge potential A. A dramatic increase of $T_{c}$ is predicted for $0.01 \mathrm{~W} \leq J \leq 10 \mathrm{~W}$. This should have immense technological impact in electric energy production, storage and transmission, as well as for medical electronics, microwave electronics, computer memory and information storage, separations technology and maglev, amongst others. The striking prediction to be confirmed by experiment is that the pairing order parameter $\Delta(\mathbf{k})$ is predicted to be $p$-wave, i.e., $l=1, S=1$, as compared to $l=2$ and $S=1$ for conventional HTS materials. In addition a novel collective model is predicted whose frequency, $\omega_{L}$ is in the optical range and is determined by $H_{s d p}$.
\end{abstract}

PACS: 71.10.Ay, 71.10.Pm.

Keywords: quantum critical point, spin fluctuation, high- $T_{C}$ superconductivity.

The static magnetic susceptibility of the localized spins in approximately 30 doped intermetallic compounds is found to be highly anomalous namely,

$$
\chi\left(\mathbf{Q}, \omega_{n}=0, T\right) \propto 1 / T^{\gamma} ; \text { with } \gamma \simeq 0.14
$$

where $\mathbf{Q}$ is the magnetic spin ordering vector of the spin ordered phase, e.g. $\mathbf{Q}=(\pi / a, \pi / a)$ for a cubic commensurate antiferromagnet. Equally anomalous is the specific heat

$$
C_{V}(T) \propto \ln T .
$$

This is to be contrasted with

$$
\chi\left(Q, \omega_{n}=0, T\right) \propto \frac{1}{T-T_{N}} \quad \text { (Néel law) }
$$

and

$$
\mathrm{C}_{V}(T) \propto T \quad \text { (Fermi liquid law) }
$$

of mean field theory. Anomalously small critical exponents are also found in some ten other experiments.
Thus, a universal experimental behavior is found, i.e., a law of corresponding states is observed, calling for a universal theory producing these anomalous exponents. Such a theory is developed here, for the first time.

In the pioneering work of Hertz [1], ferromagnetic critical spin fluctuations were treated using a one fermion loop effective action for the spin-fermion model. He found highly anomalous properties, as in experiment. This work was extended by Millis [2] to the several fermion loop level. Since then, a great deal of effort [3-9] has been expended with little progress having been achieved. Here we give a complete solution to the problem, including pairing, for the spin-fermion problem and find i) excellent agreement with experiment for the anomalous critical exponents, and ii) we predict a highly novel $p$-wave, $S=1$ superconductor, a very high frequency Leggett mode and an extraordinarily high $T_{c}$ of immense technological and scientific interest. 
For clarity, we use the spin fermion model.

$$
\begin{gathered}
H=H_{0}+H_{J}, \\
H_{0}=-\sum_{i j s} t_{i j} \psi_{i s}^{\dagger} \psi_{j s}, \\
H_{J}=J \sum_{i s s^{\prime}} \psi_{i s}^{\dagger} \psi_{i s^{\prime}} \boldsymbol{\sigma}_{s s^{\prime}} \cdot \mathbf{S}_{i},
\end{gathered}
$$

where

$$
\begin{aligned}
& \left\{\psi_{i s}^{\dagger}, \psi_{j s^{\prime}}\right\}=\delta_{i j} \delta_{s s^{\prime}} \\
& {\left[S_{i \alpha}, S_{j \beta}\right]=i \hbar S_{\gamma} \delta_{i j}}
\end{aligned}
$$

with $\alpha, \beta, \gamma=1,2,3$ in cyclic permutation. Equally well we could have use the Hubbard model with equivalent results, although the mathematics would be considerably more involved.

A key aspect of the quantum critical point problem is that all fluctuations, pairing and spin, are of long range ( $L>>a, a-$ lattice spacing), and of low frequency compared to the electronic band width $W$ and the two site Heisenberg exchange interaction, $J_{2} \simeq J^{2} / W$. This suggests that we develop a long wavelength, adiabatic approximation. This we do below.

To exploit these slow variations, we introduce the unitary electron spin rotation operator,

$$
\begin{aligned}
U(t)= & T \exp \left(\frac{i}{2} \sum_{i s s^{\prime}} \psi_{i s}^{\dagger}(t) \boldsymbol{\sigma}_{s s^{\prime}} \cdot \psi_{i s^{\prime}}(t)\right) \times \\
& \times \sin ^{-1}\left|\hat{z} \times \mathbf{S}_{i}(t)\right| \frac{\hat{z} \times \mathbf{S}_{i}(t)}{\left|\hat{z} \times \mathbf{S}_{i}(t)\right|} .
\end{aligned}
$$

$T$ is the Feynman time ordered product defined by

$$
T\left[A\left(t_{1}\right) B\left(t_{2}\right) C\left(t_{3}\right) \ldots\right]=A\left(t_{1}\right) B\left(t_{2}\right) C\left(t_{3}\right) \ldots,
$$

for $t_{1} \geq t_{2} \geq t_{3} \ldots$. This prescription preserves the quantum commutation relations (7), (8).

We define the rotation vector angle as,

$$
\boldsymbol{\Omega}_{i}(t)=\sin ^{-1}\left|\hat{z} \times \mathbf{M}_{i}(t)\right| \cdot \frac{\hat{z} \times \mathbf{M}_{i}(t)}{\left|\hat{z} \times \mathbf{M}_{i}(t)\right|}
$$

with $\mathbf{M}(\mathbf{r}, t)$ being the staggered magnetization, where for example, $\mathbf{Q}=0$ for ferromagnetic spin fluctuations. More generally,

$$
\mathbf{M}_{i}(t)=(-1)^{\mathbf{R}_{i}} \mathbf{S}_{i}(t) .
$$

The transformed Hamiltonian is

$$
\bar{H}(t)=\bar{H}_{0}(t)+\bar{H}_{J}(t)=U^{\dagger}(t)\left[H_{0}(t)+H_{J}(t)\right] U(t),
$$

where $\bar{H}_{0}$ is given for a free electron band by
$\bar{H}_{0}(t)=-\frac{\hbar^{2}}{2 m} \sum_{s s^{\prime}} d \mathbf{r}\left\{\psi_{s}^{\dagger}(\mathbf{r}, t)\left[\nabla \delta_{s s^{\prime}}+i A_{s s^{\prime}}(t)\right]^{2} \psi_{s^{\prime}}(r, t)\right\}$.

Here the gauge field $\mathbf{A}_{s s^{\prime}}(\mathbf{r}, t)$ is defined as

$$
\mathbf{A}_{s s^{\prime}}(\mathbf{r}, t)=\frac{1}{2} \boldsymbol{\sigma}_{s s^{\prime}} \cdot \nabla \boldsymbol{\Omega}(\mathbf{r}, t) .
$$

Note that $\bar{H}_{0}$ is just the electronic kinetic energy of quantum electrodynamics (QED) for $e / c \equiv 1$.

It is convenient to expand out $\bar{H}_{0}$ in powers of $\mathbf{A}$ and we find,

$$
\bar{H}_{0}(t)=H_{0}(t)+H_{s d p}(t)+H_{\text {dia }}(t)
$$

with $H_{0}(t)$ given by $(5)$. The two new spin couplings are (i) the spin deformation potential (sdp) $H_{s d p}$,

$$
\begin{gathered}
H_{s d p}(t)=\frac{i \hbar^{2}}{4 m} \int d \mathbf{r} \sum_{s s^{\prime}}\left[\psi_{s}^{\dagger}(\mathbf{r}, t) \boldsymbol{\sigma}_{s s^{\prime}} \times\right. \\
\left.\times \nabla \psi_{s^{\prime}}(\mathbf{r}, t)\right][\nabla \boldsymbol{\Omega}(\mathbf{r}, t)+\boldsymbol{\Omega}(\mathbf{r}, t) \boldsymbol{\nabla}],
\end{gathered}
$$

where the spin current is defined by

$$
\mathbf{j}_{s}(\mathbf{r}, t) \equiv-\frac{i \hbar}{2} \sum_{s s^{\prime}} \psi_{s}^{\dagger}(\mathbf{r}, t) \nabla \psi_{s^{\prime}}(\mathbf{r}, t) \sigma_{s s^{\prime}}
$$

$\mathbf{j}_{S}(\mathbf{r}, t)$ couples to the quantity $\nabla \boldsymbol{\Omega}(\mathbf{r}, t)$, in the same manner as the $\mathbf{p} \cdot \mathbf{A}+\mathbf{A} \cdot \mathbf{p}$ paramagnetic coupling of QED. The «diamagnetic» term proportional to $\mathbf{A}(\mathbf{r}, t)^{2}$ is given by

$$
H_{\text {dia }}(t)=-\frac{\hbar^{2}}{8 m} \int_{|\nabla \Omega(\mathbf{r}, t)|^{2}} d \mathbf{r} \sum_{s} \psi_{s}^{\dagger}(\mathbf{r}, t) \psi_{s}(\mathbf{r}, t) .
$$

It is $H_{s d p}$ and $H_{\mathrm{dia}}$ which lead to the anomalous exponents near the QCP.

$\bar{H}_{J}(t)$ is given by

$$
H_{J}(t)=\frac{J}{2} \int d \mathbf{r} \sum_{\bar{s}} \bar{s} \psi_{\bar{S}}^{\dagger} \psi_{\bar{S}}(\mathbf{r}, t)|S(\mathbf{r}, t)|(-1)^{r},
$$

where $\bar{s}$ is defined as the $\hat{z}$ in the spin rotated frame. We note that the transformed exchange coupling acts as a simple diagonal spin Zeeman coupling, analogous to the exchange interaction in the mean field approximation. This exact result greatly simplifies the calculations.

While the discussion at this point is exact, it is helpful to make pairing correlations manifest by elevating $\psi_{i s}^{\dagger}(t)$ to a two component pseudo-spin operator,

$$
\psi_{i s}^{\dagger}(t)=\left[\psi_{i \uparrow}^{\dagger}(t), \psi_{i \downarrow}^{\dagger}(t)\right] .
$$

We introduce the Pauli pseudo-spin matrices $\tau_{i}$, with $i=0,1,2,3$. Then $\bar{H}(t)$ can be written as 


$$
\begin{aligned}
\bar{H}_{0}(t)= & -\frac{\hbar^{2}}{2 m} \sum_{s^{\prime} s} \int d \mathbf{r} \Psi_{s}^{\dagger}(\mathbf{r}, t)\left[\nabla \tau_{3} \delta_{s s^{\prime}}+\right. \\
& \left.+i \mathbf{A}_{s s^{\prime}}(\mathbf{r}, t)\right]^{2} \Psi_{s^{\prime}}(\mathbf{r}, t)
\end{aligned}
$$

and

$$
\bar{H}_{J}(t)=J \sum_{\bar{s}} \Psi_{\bar{s}}^{\dagger}(\mathbf{r}, t) 1 \Psi_{\bar{S}} S_{\hat{z}}(t),
$$

where $\bar{s}$ and $\hat{z}$ correspond to the direction of the local $\mathbf{M}(\mathbf{r}, t)$. The one loop Gor'kov self energy is given by

$$
\Sigma\left(\mathbf{k}, \omega_{n}\right)=\sum_{\mathbf{Q}, \omega_{m}}\left[V\left(Q, \omega_{n}-\omega_{m}\right) G\left(k-Q, \omega_{n}-\omega_{m}\right)\right]
$$

where $\omega_{n}=(2 n+1) k_{B} T, \omega_{m}=2 \pi m k_{B} T$ and $V$ is the potential arising from $\bar{H}_{s d p}$ and $\bar{H}_{\text {dia }}$. Taking the 1,2 components of (22) we have the gap equation,

$\Sigma\left(k, \omega_{n}\right)_{12}=\sum_{\mathbf{Q}, \omega_{n}}\left[V\left(Q, \omega_{n}-\omega_{m}\right) G\left(k-Q, \omega_{n}-\omega_{m}\right)\right]_{12}$.

The normal phase renormalization shifts are given by the 1 and $\tau_{3}$ terms in (22), respectively.

While $\bar{H}_{\text {dia }}$ and $\bar{H}_{J}$ give $s$-wave $(l=0)$ and $d$-wave $(l=2)(S=0)$ pairing, $T_{C}$ is exponentially larger for $p$-wave, $(l=1),(S=1)$ pairing. To calculate the effective pairing interaction in the $p$-wave, $S=1$ channel we introduce quasi particles which diagonalize $H_{0}(t)$ and $\bar{H}_{J}(t)$. These terms in $H$ couple each $k$ with $k \pm Q$ in reciprocal space, without spin flip. Introducing the Bogoliubov-Valatin quasi-particle transformation, one has

$$
\gamma_{k, s}=u_{k} \psi_{k s}+v_{k s} \psi_{k+Q_{s}}
$$

and

$$
\gamma_{k+Q, s}=v_{k s} \psi_{k s}-u_{k} \psi_{k+Q, s}
$$

with eigenvalues

$$
\pm E_{k}= \pm \sqrt{\epsilon_{k}^{2}+(J / 2)^{2}} .
$$

Here,

$$
\begin{aligned}
& u_{k s} \equiv \sqrt{1 / 2\left(1+\epsilon_{k} / E_{k}\right)}, \\
& v_{k s} \equiv \sqrt{1 / 2\left(1-\epsilon_{k} / E_{k}\right)} .
\end{aligned}
$$

In the $\gamma$ representation, $H_{s d p}(t)$ becomes
$H_{s d p}(t)=-\frac{i \hbar^{2}}{4 m} \sum_{s s^{\prime}} \gamma_{s}^{\dagger}(\mathbf{r}, t) 1 \gamma_{s^{\prime}}(\mathbf{r}, t)(\nabla \boldsymbol{\Omega}(\mathbf{r}, t)+\boldsymbol{\Omega}(\mathbf{r}, t) \nabla)$

with

$$
E_{k}=\sqrt{\left(\frac{\hbar^{2} k^{2}}{2 m}-\mu\right)^{2}+\frac{J^{2}}{4}}
$$

for the free electron model.

The ground canonical potential is given by,

$\Lambda(T)=-k_{B} T \ln \operatorname{Tr} T \exp (-\beta(\bar{H}-\mu N))=-k_{B} T \ln Z(T)$.

Within the RPA for $\bar{H}_{s d p}(t)$ and $\bar{H}_{\text {dia }}(t)$, as shown in Fig. 1, one has,

$$
\begin{gathered}
\Lambda(T)_{R P A}=\sum_{\mathbf{Q}, \omega_{m}} \operatorname{Tr}\left\{V\left(\mathbf{Q}, \omega_{n}\right) \Phi_{0}\left(\mathbf{Q}, \omega_{n}\right) \times\right. \\
\left.\times\left[1-V\left(\mathbf{Q}, \omega_{n}\right) \Phi_{0}\left(\mathbf{Q}, \omega_{n}\right)\right]^{-1}\right\},
\end{gathered}
$$

where the zero order irreducible polarizability is defined by

$$
\Phi_{0}\left(\mathbf{Q}, \omega_{m}\right)=\sum_{k, \omega_{n}} G_{0}\left(\mathbf{k}+\mathbf{Q}, \omega_{n}+\omega_{m}\right) G_{0}\left(\mathbf{k}, \omega_{n}\right) .
$$

The specific heat is given by

$$
C_{V}(T) \equiv-\frac{d}{d T} k_{B} T \ln Z(T) .
$$

We find $C_{V}(T) \simeq \ln T$ for $W / 100<T<W$, as obsereved in experiment.

The RPA spin susceptibility is given by

$$
\begin{gathered}
\chi_{R P A}\left(Q, \omega_{n}, T\right)=\frac{1}{4} \sum_{s \bar{s}} s \bar{s} \Phi_{0}\left(Q, \omega_{n}, T\right) \times \\
\times\left[1-V\left(Q, \omega_{n}, T\right) \Phi_{0}\left(Q, \omega_{n}\right)\right]^{-1} .
\end{gathered}
$$

To estimate $T_{c}$, we use a square potential model, as in the BCS model. Then

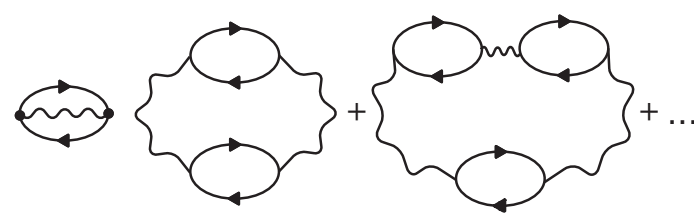

Fig. 1. The grand canonical $\Lambda(T)$ within the random phase approximation. 


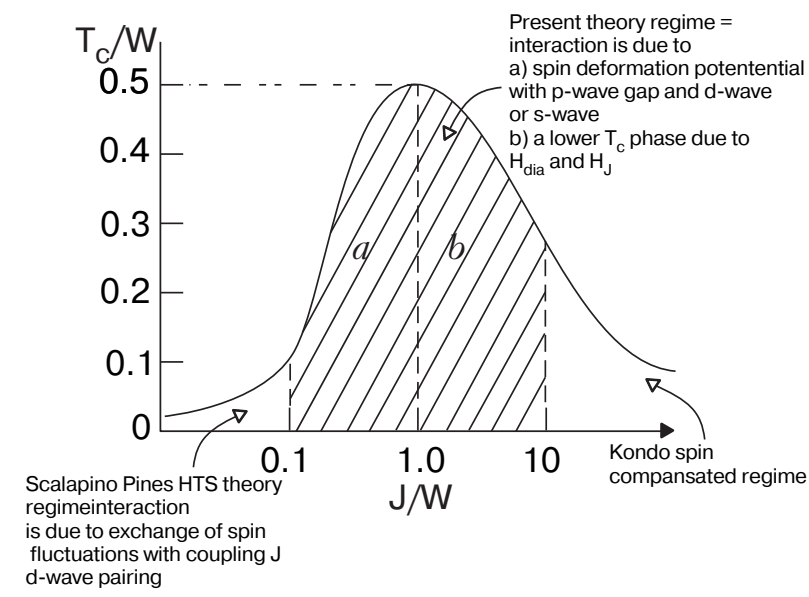

Fig. 2. The phase diagram of the $t-J$ model, showing the conventional nearly antiferromagnetic Fermi liquid of Pines and Scalapins for $J \leq 0.01 W$, where $J$ is the electron localized spin exchange coupling and $W$ is the electronic band width. For $0.01 W \leq J \leq 10 W$ a novel $p$-wave, $l=1, s=1$ phase is predicted with an extremely high $T_{c}$ of immense technological importance (see the text). In this phase the existence of a Leggett collective model is predicted, corresponding to an oscillation at frequency ${ }^{\circ} L$ of the angle between $\mathbf{L}$ and $\mathbf{s}$ vectors the pair. However, here the novel strong spin deformation raises ${ }^{{ }^{10}} L$ to a high value of order the optical range vs the low frequency of superfluid ${ }^{3} \mathrm{He}$, where the spin orbit coupling $H_{s o}$ is extremely weak.

$$
T_{c}=1.14 \omega_{s} \mathrm{e}^{-\frac{1+\lambda}{\lambda}}
$$

where

$$
\lambda=N(0) V_{\text {pair }},
$$

and $V_{\text {pair }}$ being given by (22).

To determine $T_{c}$ we take the limit that the magnitude of the order parameter $\Delta\left(k, \omega_{n}, T\right)$ goes to zero. One finds that $T_{c}$ satisfies the linear gap equation

$$
\begin{gathered}
\Delta\left(\mathbf{k}, \omega_{n}, T_{c}\right)=-\sum_{Q, \omega_{m}} \frac{1}{Z\left(\mathbf{k}, \mathbf{Q}, \omega_{m}\right)}\left(u_{k} v_{k+Q}+v_{k} u_{k Q}\right)^{2} \times \\
\times V\left(\mathbf{k}, \mathbf{Q}, \omega_{m}, T\right) G_{0}\left(k+Q, \omega_{n}+\omega_{m}\right) \times \\
\times G_{0}\left(-\mathbf{k}-\mathbf{Q},-\omega_{n}-\omega_{m}\right) \Delta\left(\mathbf{k}+\mathbf{Q}, \omega_{n}+\omega_{m}, T\right),
\end{gathered}
$$

where $Z\left(k, Q, \omega_{m}\right) / \omega_{n}$ is the parallel component of $\Sigma$ (see (22)). The quantity $\left(u v^{\prime}+v u^{\prime}\right)$ is nothing but the BCS coherence. For the BCS square potential in energy, with a cut off at spin fluctuation frequency $\omega_{s}$,

$$
V\left(\mathbf{k}, \mathbf{Q}, \omega_{m}, T\right)=V_{0}(\mathbf{k}, Q, T), \quad\left|\omega_{m}\right| \leq \omega_{s},
$$
one finds

$$
k_{B} T_{c} \simeq \omega_{s} \mathrm{e}^{-\frac{1+\lambda}{\lambda}}
$$

where the coupling constant $\lambda$ is given by

$$
\lambda=N(0)\left(\frac{\hbar^{2}}{4 m}\right)^{2}\left[\frac{\left|V_{0}\left(Q_{0}, T\right)\right|^{2}}{\omega_{s}}\left\langle\left(u_{k} v_{k+Q}+v_{k} u_{k+Q}\right)^{2}\right\rangle\right]
$$

Using

$$
\omega_{s} \simeq \frac{J^{2}}{W},
$$

we obtain

$$
k_{B} T_{c} \simeq \frac{J^{2}}{W} \mathrm{e}^{-\frac{1+\lambda}{\lambda}}
$$

Maximizing $T_{c}$ one finds

$$
k_{B} T_{c} \simeq \frac{W}{8}
$$

for $J \simeq 0.1 W$. For $W \simeq 10 \mathrm{eV}$, this leads to the remarkably high value of

$$
k_{B} T_{c} \simeq 1 \mathrm{eV} \simeq 10^{4} \mathrm{~K}
$$

with $J=1 \mathrm{eV}$. Clearly this result is of immense technological as well as scientific value (Fig. 2).

Details of this new theory with applications to electronic tunneling, ARPES, transport measurement, magnetic penetration depth, $C_{V}(T)$, etc. will soon be published elsewhere (PR).

The author wishes to thank Profs. Megan Aronson, Nicholas Bonesteel, Lev P. Gor'kov and Kun Yang for helpful discussions. This work was supported in part by a grant from the National Science Foundation, grant No. 0084173 and Department of Energy, grant No. DE-FG03-03NA00066. The author would like to express his sincere thanks to Layla Hormozi for help with preparation of the manuscript.

1. J.A. Hertz, Phys. Rev. B14, 1165-1184 (1976).

2. A.J. Millis, Phys. Rev. B48, 7183 (1993).

3. A.V. Chubukov and D.L. Maslov, Phys. Rev. B68, 155113 (2003)

4. P. Schlottmann, Phys. Rev. B68, 125105 (2003).

5. Q. Si, S. Rabello, K. Ingersent, and J.L. Smith, Phys. Rev. B68, 115103 (2003).

6. S. Sachdev and T. Morinari, Phys. Rev. B66, 235117 (2002).

7. Z. Wang, W. Mao, and K. Bedell, Phys. Rev. Lett. 87, 257001 (2001).

8. V.P. Mineev and M. Sigrist, Phys. Rev. B63, 172504 (2001).

9. M.J. Lercher and J.M. Wheatly, Phys. Rev. B63, 12403 (2001). 